Research Article

\title{
Protective Effect of Ultraviolet C Irradiation on the Gastric Mucosa of Rats with Chronic Gastritis Induced by Physicochemical Stimulations
}

\author{
Likang Wang $\mathbb{D}^{1,2}$ Wei Chen, ${ }^{3}$ Xisheng Lin, ${ }^{3}$ Zhao Zhang, ${ }^{3}$ Na Wang, ${ }^{3}$ Youkui Lv, ${ }^{1}$ \\ Xinglin Wang $\left(\mathbb{D},{ }^{1}\right.$ and Yueming Gao $\mathbb{1}^{3}$ \\ ${ }^{1}$ Medical School of Chinese PLA, Beijing 100853, China \\ ${ }^{2}$ Department of Rehabilitation Medicine, The Third Medical Centre, Chinese PLA General Hospital, Beijing 100039, China \\ ${ }^{3}$ Department of Rehabilitation Medicine, The Second Medical Centre, Chinese PLA General Hospital, Beijing 100853, China
}

Correspondence should be addressed to Xinglin Wang; wangxl30188@163.com and Yueming Gao; gaoym301@sina.com

Received 21 May 2020; Revised 12 February 2021; Accepted 9 March 2021; Published 17 March 2021

Academic Editor: Muhammad Jahangir Hossen

Copyright (C) 2021 Likang Wang et al. This is an open access article distributed under the Creative Commons Attribution License, which permits unrestricted use, distribution, and reproduction in any medium, provided the original work is properly cited.

Background. Chronic gastritis (CG) is a common digestive disease with the highest morbidity among multiple digestive diseases, which seriously lowers the life quality of patients. The pathological alternations of gastric mucosa, and its possible mechanisms have been the focus of CG-related researches. Accumulative basic and clinical evidence has confirmed that ultraviolet C (UVC) is effective in relieving superficial acute infective inflammation, skin and mucous membrane injuries, and ulcers, and promoting wound healing. Objective. This study was aimed at investigating the protective effects of UVC on gastric mucosal injury in rats stimulated with physicochemical irritants like ethanol and exploring the mechanisms underlying the protection by UVC against gastric mucosal injury and CG. Methods. Fifty Wistar rats were randomly divided into five groups, including Group A (normal), Group B (model), Group C (omeprazole treatment), Group D (intragastric UVC irradiation for $24 \mathrm{~s} \times 2$ yields), and Group E (intragastric UVC irradiation for $48 \mathrm{~s} \times 2$ yields). Rats in Groups B-E were made CG model by physicochemical stimulations. All rats were sacrificed one week after the 22-week experiment, and gastric tissues were harvested. Histopathological examinations were performed. The activities of superoxide dismutase and catalase as well as the contents of reduced glutathione and malondialdehyde in gastric mucosal tissues were detected. Serum interleukin-6, interleukin-1beta, tumor necrosis factor-alpha, pepsin, and gastrin were measured. Results. Results showed that physiochemical irritants like ethanol could be used for easily establishing a rat CG model that shared similar pathological features with human CG. Intragastric UVC irradiation could promote the repair of gastric mucosa and improve the atrophy of gastric mucosa by inhibiting the inflammatory factors, increasing the levels of pepsin and gastrin, decreasing the expression of lipid peroxide, and enhancing the activity of superoxide dismutase and catalase and the levels of reduced glutathione. UVC irradiation for $48 \mathrm{~s} \times 2$ yields showed the strongest protective effect. Conclusion. UVC irradiation could inhibit the inflammatory factors, activate the antioxidative system, and enhance the secretion of pepsin and gastrin, which promoted the repair of injured gastric mucosa and improved gastric mucosa atrophy.

\section{Introduction}

Chronic gastritis (CG), which is featured by complicated pathophysiological processes, refers to chronic gastric mucosal injury caused by the imbalance between aggressive and defensive factors. The common pathogenic elements include Helicobacter pylori (Hp), alcohol, drugs, duodenogastric reflux, duodenal fluid, autoimmunity, etc. [1]. Currently, the occurrence of CG is considered to be correlated with decreased pepsin and gastrin and enhanced oxidative stress, but the exact pathogenesis remains unclear. Clinically, few specific therapeutic agents are available for CG, while monotherapy or combination therapy like acid suppression, promotion of gastric motility, protection of gastric mucosa, and antibiotics are accepted as general CG therapies. In most cases, these treatments can effectively relieve the symptoms 
of CG for a relatively long period, but they generally have few therapeutic effects on gastric mucosal atrophy and several of them could induce gastric dysfunction, injury, and even cancer [2]. In addition, disease recurrence after drug withdrawal, antibiotic resistance after long-term medication, and several other side effects complicate the treatment of CG [3-5]. Long-term nonatrophic CG is very likely to progress into atrophic CG, which is a recognized precursor of gastric cancer [6].

Ultraviolet C (UVC) irradiation is a common physiotherapy with recognized clinical effectiveness. UVC can significantly reduce superficial aseptic inflammation, mucosal inflammation, and ulcers and promote tissue healing [7-11].

With the invention and application of endoscopic technology, plentiful physical factors, such as microwave, laser, and ultrasound, have been applied to treat tissues and organs inside the human body. This technology also promotes the application of UVC in treating digestive diseases like CG by irradiating UVC into the gastric cavity through the esophagus with the assistance of endoscope.

In this study, we postulated that UVC irradiation could ameliorate CG and validated our hypothesis by establishing a CG animal model. The possible mechanism was also discussed.

We verified our conjecture by detecting interleukin-6 (IL-6), interleukin-1beta (IL-1 $\beta$ ), tumor necrosis factoralpha (TNF- $\alpha$ ), pepsinogens (PGI, PGII, PGR), gastrin-17 (G-17), reduced glutathione (GSH), catalase (CAT), malondialdehyde (MDA), and superoxide dismutase (SOD), and discussed its mechanism.

\section{Materials and Methods}

2.1. UVC Dose. KDY-1 UVC apparatus was produced by the First Medical Center of PLA General Hospital and Beijing Keda Light Source Electric Appliances Co., Ltd. The wavelength ranged from 230 to $280 \mathrm{~nm}$ with a $90 \%$ percentage of $253.7 \mathrm{~nm}$. The surface power was $1 \mathrm{~mW}$. The quartz derivation was $12 \mathrm{~cm}$ long and $3 \mathrm{~mm}$ in diameter with a semicircular head.

2.1.1. Detection of UVC Output Dosage. The detection of UVC output dosage was divided into five steps. Firstly, the UV meter was turned on, the wavelength response was set as $254 \mathrm{~nm}$, and the range factor was set as 100. Secondly, the photosensitive surface of the probe was placed in the position to be tested. Thirdly, the UVC apparatus was turned on, the optical output mode was selected, and preheating occurred for $30 \mathrm{sec}$ to stabilize the output dose. Fourthly, the optical head was attached to the light-sensitive probe, UVC exported, the test run for $10 \mathrm{sec}$, and the test value recorded. Fifthly, the power density was calculated according to the formula: power density $\left(\mathrm{mW} / \mathrm{cm}^{2}\right)=$ test value $\left(\mu \mathrm{W} / \mathrm{cm}^{2}\right) \times$ range factor $(100) / 1000$.

2.1.2. Test Results. Results were obtained from triplicate experiments. The diameter of the optical head was $3 \mathrm{~mm}$.
The area of the optical head was $0.07 \mathrm{~cm}^{2}$. According to the formula used in the calculation of clinical biological doses, the output dose of an optical head with an area of $0.34 \mathrm{~cm}^{2}$ reaching over $60 \mathrm{~mW} / \mathrm{cm}^{2}$ is defined as the $1 \mathrm{sec}$ minimal erythema dose (MED). It could be inferred that the output dose of the optical head with an area of $0.07 \mathrm{~cm}^{2}$ was $4.1 \mathrm{~mW} / \mathrm{cm}^{2}$, which was equal to $1 / 3 \mathrm{MED}$.

\subsubsection{Determination of UVC Dose for the Treatment of CG.} The common dose that is used for the clinical treatment of oral mucosal injury or ulcer is $8 \mathrm{MED}[12,13]$. Moreover, direct exposure of the optical conductor to the damage surface is required, and radiancy will decrease by $50 \%$ when the exposure distance reaches $1 \mathrm{~cm}$ away from the damage surface. In this study, UVC irradiation was performed blindly within rat gastric tissues with smaller exposure area than the straight optical conductor. The presence of gastric juice also prevented the irradiation from reaching the mucosal surface precisely. Gastric mucosal injury can occur in both gastric antrum and body. Accordingly, irradiation for 2 yields was selected in this study, including 1-yield irradiation at the gastric fundus and 1-yield irradiation at $1 \mathrm{~cm}$ from the gastric fundus. Thus, based on the previous animal experiments and the preliminary results of our team, we finally chose $24 \mathrm{~s} \times 2$ yields and $48 \mathrm{~s} \times 2$ yields for the treatment of CG in this study $[14,15]$.

\subsection{Establishment of Chronic Gastritis Model in Rats}

2.2.1. Animals. Fifty healthy SPF Wistar rats (male: female $=1: 1,8$ weeks old), weighing $200 \pm 20 \mathrm{~g}$, were purchased from Medical Animal Laboratory Center, The First Medical Center of PLA General Hospital (certificate number: SCXK (Beijing) 2019-0009). All rats were housed in cages and placed in a clean room with temperatures of $20-23^{\circ} \mathrm{C}$, humidity of $50 \%-60 \%$, and a $12 \mathrm{~h}$ dark/light cycle. During the experiment, rats had free access to chow diet and water. The study was conducted under the guidance of the National Regulations Affairs Concerning Experimental Animals and approved by the Ethics Committee of the First Medical Center of PLA General Hospital (No. 2018pkyn049).

2.2.2. Reagents and Chemicals. All chemicals used to induce CG and treatment were obtained from Aoboxing BioTechnology Co., Ltd. (Beijing, China). The rat immunosorbent rat assay (ELISA) kits of cytokines (IL-6, IL-1 $\beta$, and TNF- $\alpha$ ), pepsinogens (PGI, PGII), gastrin-17 (G-17), reduced glutathione (GSH), catalase (CAT), malondialdehyde (MDA), and superoxide dismutase (SOD) were all supplied by Jianglai Bio-Technology Co., Ltd. (Shanghai, China). All the chemicals used in the experiment were of reagent grade unless otherwise stated.

2.2.3. Experimental Grouping and Establishment of CG Model in Rats. After the two-week adaptive feeding, fifty rats were randomly divided into five groups (10 rats per 
group), including normal group (Group A), model group (Group B), $20 \mathrm{mg} / \mathrm{kg}$ omeprazole (OMEP) (Group C), $24 \mathrm{~s}$ intragastric UVC irradiation group (Group D), and $48 \mathrm{~s}$ intragastric UVC irradiation group (Group E). The rats in Group A had free access to chow diet and water. Each rat in Groups B-E was intragastrically administrated with $1 \mathrm{~mL}$ anhydrous ethanol weekly for eight weeks and had free access to sodium deoxycholate solution (2\%) in place of water. In the next fourteen weeks, these rats were given sodium deoxycholate solution (2\%), ethanol (20\%), or ammonium hydroxide $(0.1 \%)$ alternately every two weeks $[16,17]$. The rats in Group A were administered with water instead of anhydrous ethanol by gavage.

A stainless steel needle with a diameter of $45 \mathrm{~mm}$ was used for gavage. Water bottles were routinely cleaned, and the liquids in the bottles were changed daily. All the rats were fasted and dehydrated for $12 \mathrm{~h}$ before and after each gavage or irradiation.

2.3. Interventions. All rats had free access to chow diet and water after the successful establishment of the CG model. All the experiments were performed in the morning. All the rats were fasted and dehydrated for $12 \mathrm{~h}$ before each experiment.

Group A: intragastric intubation using the quartz derivation without irradiation for seven constructive days

Group B: no interventions

Group C: OMEP $(20 \mathrm{mg} / \mathrm{kg})$ for seven constructive days [18]

Group D: intragastric UVC irradiation for $24 \mathrm{~s} \times 2$ yields on day 1 and intubation without irradiation on day 2 to day 7

Group E: intragastric UVC irradiation for $48 \mathrm{~s} \times 2$ yields on day 1 and intubation without irradiation on day 2 to day 7

All rats had free access to chow diet and water after the whole experiment and fasted and dehydrated for $12 \mathrm{~h}$ at one week later. Then, these rats were anesthetized by injection with $3 \%$ pentobarbital sodium solution $(45 \mathrm{mg} / \mathrm{kg})$. Incisions were made downward $4-5 \mathrm{~cm}$ from the xiphoid and abdominal aorta was exposed. Blood was collected by puncture from distal to the proximal abdominal aorta and stored in blood-collecting tubes. Sera were collected from whole blood after centrifugation at $3000 \mathrm{rpm}$ for $15 \mathrm{~min}$ and stored at $-80^{\circ} \mathrm{C}$ for further analyses. Rats were then sacrificed and gastric tissues were collected and preserved in $-80^{\circ} \mathrm{C}$ for examination of GSH, CAT, SOD, and MDA.

2.4. Gastric Histopathological Examination. Rat gastric tissues were sheared along the greater gastric curvature and gently washed with flowing normal saline. The tissue specimens were fixed in 10\% formaldehyde solution for $48 \mathrm{~h}$ and then dehydrated with gradient alcohol. Then, the specimens were cleared with xylene and immersed and embedded with paraffin.
Four micron thick gastric tissue slices were prepared and stained with hematoxylin and eosin (H\&E) using standard methods. The thickness of the gastric wall, the color and luster of gastric mucosa, the number and elasticity of mucosal folds, edema, and gastrorrhagia were observed and recorded. The activity of gastric mucosa, chronic inflammatory response, and gastric mucosal atrophy were classified into four grades based on the degrees of neutrophil infiltration, including normal, mild, moderate, and severe [19]. The four grades were scored as $0(0=$ normal $), 1$ $(1=$ mild $), 2$ ( 2 = moderate), and 3 ( 3 = severe), respectively; then, the scores of gastric mucosal activity (scored by neutrophil infiltration in the setting of a chronic inflammatory response), chronic inflammatory response (graded according to the density and depth of the infiltration of chronic inflammatory response cells into the mucosal layer, and under ambiguous conditions, the classification should be based on the cell density), and gastric mucosal atrophy (diagnosed by a $1 / 3$ reduction in the gastric intrinsic glands) were calculated and defined as the indicators for evaluating the efficacies of treatments $[20,21]$. The pathological evaluation was performed by two pathologists with more than 10-year experience.

\subsection{Determination of Serum Interleukin-6 (IL-6), Interleukin-} 1 Beta (IL-1 $\beta$ ), and Tumor Necrosis Factor-Alpha (TNF- $\alpha$ ) Levels. The levels of IL-6 (JL20896), IL-1 $\beta$ (JL20884), and TNF- $\alpha$ (JL13202) in serum were determined by rat ELISA kits, and the procedures of IL- 6, IL- $1 \beta$, and TNF- $\alpha$ kits were described in [22].

2.6. Measurement of Serum Pepsinogens (PGI, PGII), PGR (PGI/PGII), and Gastrin-17 (G-17) levels. The levels of serum PGI (JL21317), PGII (JL19874), and G-17 (JL45791) were detected using rat ELISA kits [23]. PGR is the ratio of PGI and PGII. According to the instructions, the OD value at $450 \mathrm{~nm}$ of the supernatant was quantitatively determined using the PGI, PGII, and G-17 kits.

2.7. Detection of GSH, CAT, SOD, and MDA Levels in Rat Gastric Tissues. The activities of SOD (JL22893) and CAT (JL21028) as well as the contents of GSH (L21015), MDA (JL13297), and proteins were measured for evaluating the degrees of lipid peroxidation in rat gastric tissues and detected using rat ELISA kits [24]. Briefly, rat gastric tissues were washed using normal saline and then dried out with a towel. The loading buffer was added based on sample weight. Then tissues were homogenated using a Teflon Homogenizer and centrifuged at $4^{\circ} \mathrm{C}, 10,000 \mathrm{rpm}$ for $60 \mathrm{~min}$, which was stored in a refrigerator at $-80^{\circ} \mathrm{C}$ before experiments. The supernatant was separated for measuring GSH, CAT, SOD, and MDA according to the manufacturer in the kits. The OD values were detected at $450 \mathrm{~nm}$ using a spectrophotometer.

2.8. Statistical Analysis. For the quantitative data, data are expressed as median, quartile range ( $\mathrm{M}, \mathrm{QR})$. The differences between groups were compared by one-way ANOVA using 
SPSS software ver.19 (IBM-SPSS Inc., Chicago, IL, USA). Data are expressed as mean \pm standard deviation. Values of $P<0.05$ were considered statistically significant.

\section{Results}

3.1. Establishment of the CG Model in Rats. Three rats died during the establishment of the model (Table 1). The rat in Group B died possibly from the improper experimental operation. The rat struggled hard when grasped by the researcher. Then, its body below its head and neck escaped from the researcher's hands, which caused its death from cervical dislocation. The esophagus of the rat in Group C was punctured by the rough lavage needle and fluid flowed into its trachea. Then, the rat died because of asphyxia. The rat in Group E developed body emaciation and hypotrichosis when it died. Gastric dissection showed severe hyperemia and erosions in the gastric mucosa and disappeared plica mucosa. This could be a result of the model establishment.

3.2. UVC Irradiation Relieves Gastric Mucosal Lesions of CG Rats. Most CG rats were characterized by the uneven inelastic gastric wall, pale or dark mucosa, with a small amount of mucus adhered, and disorganized, scarce, or flat gastric folds. Some of the rats developed gastric mucosal hemorrhage and/or edema. Results showed that gastric mucosal injury was significantly relieved in rats which had received UVC irradiation for $48 \mathrm{~s} \times 2$ yields. UVC irradiation for $48 \mathrm{~s} \times 2$ yields was comparable to OMEP in improving gastric mucosal injury. Macroscopic and histopathological examinations revealed that both UVC irradiations for $24 s \times 2$ yields and $48 s \times 2$ yields effectively mitigated ethanol-induced gastric mucosal injury in CG rats, while UVC irradiation for $48 \mathrm{~s} \times 2$ yields showed a greater efficacy.

It is of great importance for the evaluation and quantification of inflammation and atrophy during CG to determine the degrees of gastric mucosal activity, chronic inflammatory response, and gastric mucosal atrophy. As shown in Figures 1 and 2 and Table 2, both OMEP administration and UVC irradiation could significantly decrease the degrees of gastric mucosal activity (Figure 2(a), Table 2), chronic inflammatory response (Figure 2(b), Table 2), and gastric mucosal atrophy (Figure 2(c), Table 2). The effects of UVC irradiation for $24 \mathrm{~s} \times 2$ yields were comparable to those of OMEP, while the effects of UVC irradiation for $48 \mathrm{~s} \times 2$ yields were the strongest.

3.3. UVCIrradiation Decreases the Levels of Serum IL-6, IL-1 $\beta$, and TNF- $\alpha$ in CG Rats. The serum levels of IL-6 (Figure 3(a)), IL-1 $\beta$ (Figure 3(b)), and TNF- $\alpha$ (Figure 3(c)) were determined. The levels of TNF- $\alpha$ and IL- $\beta$ in model group were higher than those in normal group. $24 \mathrm{~s} \times 2$ field irradiation and OMEP had the same effect in reducing the levels of IL-6, IL- $1 \beta$, and TNF- $\alpha$, while $48 \mathrm{~s} \times 2$ field irradiation had the most significant effect.
TABLe 1: The mortality of CG rats.

\begin{tabular}{lcc}
\hline Groups & $n$ & Mortality \\
\hline Normal & 10 & 0 \\
Model & 10 & 1 \\
OMEP & 10 & 1 \\
Ultraviolet C $(24 \mathrm{~s} \times 2)$ & 10 & 0 \\
Ultraviolet C $(48 \mathrm{~s} \times 2)$ & 10 & 1 \\
\hline
\end{tabular}

One CG model rat, one OMEP-treated rat, and one rat receiving ultraviolet $\mathrm{C}$ irradiation for $48 \times 2$ yields died during the experiment.

3.4. UVC Irradiation Decreases the Levels of Serum PGI, PGII, $P G R$, and G-17 in CG Rats. The levels of serum PGI, PGII, PGR, and G-17 in all rats were measured (Figure 4). Results showed that UVC irradiation had no effects on the level of serum PGII but increased the levels of serum PGI, PGR, and G-17 in CG rats. This could be explained by the different sources and distribution of PGII [25]. The increase in serum PGI levels caused an increase in serum PGR levels. UVC irradiation for $24 \mathrm{~s} \times 2$ yields showed equivalent effects to OMEP on increasing the levels of serum PGI, PGR, and G-17 in CG rats. UVC irradiation for $48 \mathrm{~s} \times 2$ yields exhibited the strongest ameliorative effect.

3.5. Effects of UVC on GSH, CAT, SOD, and MDA in Gastric Tissues of Rats with CG. Generally, oxidant and antioxidant systems in organisms are kept under a dynamic balance. However, the development of CG is usually accompanied by enhanced oxidative stress and damaged antioxidant system in gastric tissue [26, 27]. MDA is a biomarker of oxidative stress. GSH, CAT, and SOD eliminated intracellular oxygen free radicals in cells, thereby protecting cells from injury [28]. Herein, we measured the levels of GSH, CAT, SOD, and MDA in rat gastric tissues (Figure 5). Results showed that OMEP significantly increased the activity of CAT and SOD as well as GSH levels and decreased the content of MDA possibly by reducing the secretion of gastric acid and promoting the repair of the gastric mucosa UVC irradiation for $24 s \times 2$ yields had comparable effects to OMEP on increasing CAT and SOD activity, improving GSH levels, and decreasing MDA content, while UVC irradiation for $48 \mathrm{~s} \times 2$ yields showed a stronger antioxidative effect.

\section{Discussion}

The mechanisms of UVC promoting tissue regeneration remained unclear. The existing literature showed that, in addition to the direct disinfection and sterilization effects on local lesions, appropriate UVC irradiation could accelerate and promote tissue repair, including skin, mucosa, and other soft tissues. For instance, short-time low-dose UVC irradiation could induce the photochemical reaction on the skin, enhance cellular metabolism, improve tissue vasospasm, accelerate blood flow, and increase blood perfusion [7]. UVC could also facilitate the synthesis of DNA and RNA and promote mitosis, cell growth, and reproduction. In addition, UVC could stimulate cells to produce and secrete various cytokines, such as IL-6, IL- $1 \beta$, and TNF- $\alpha$, etc. Through the chemotaxis of the cytokines above, granulocytes, mononuclear macrophages, and 


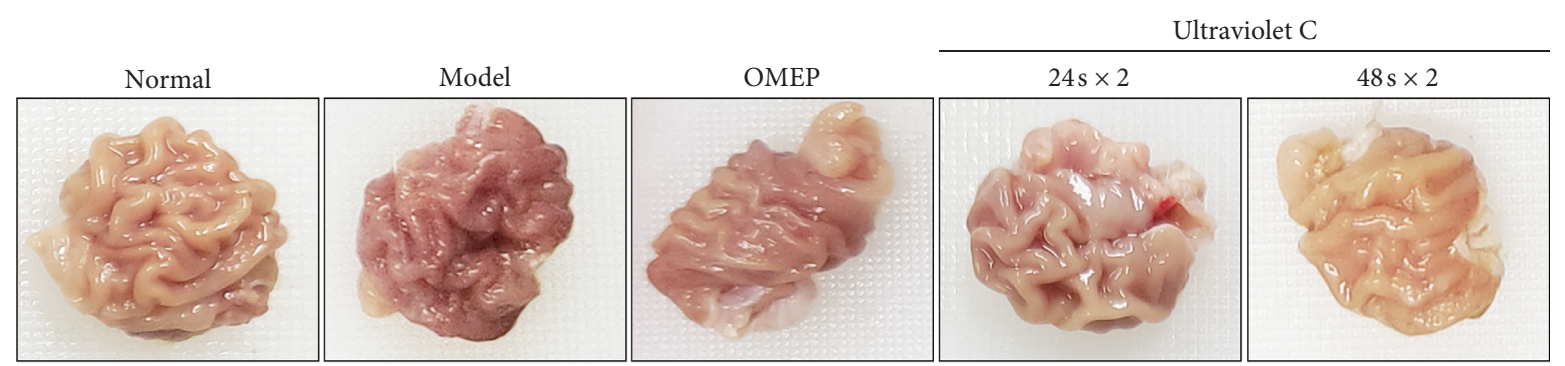

(a)

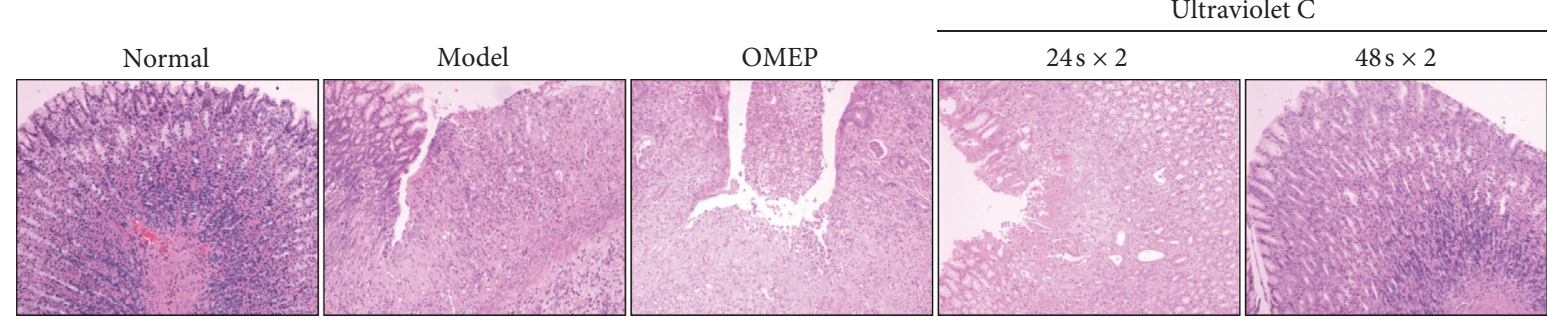

(b)

FIGURE 1: Effect of UVC irradiation on gastric tissues of rats with CG. (a) Gastric morphology. (b) HE staining of the gastric mucosa ( $\times 100)$.

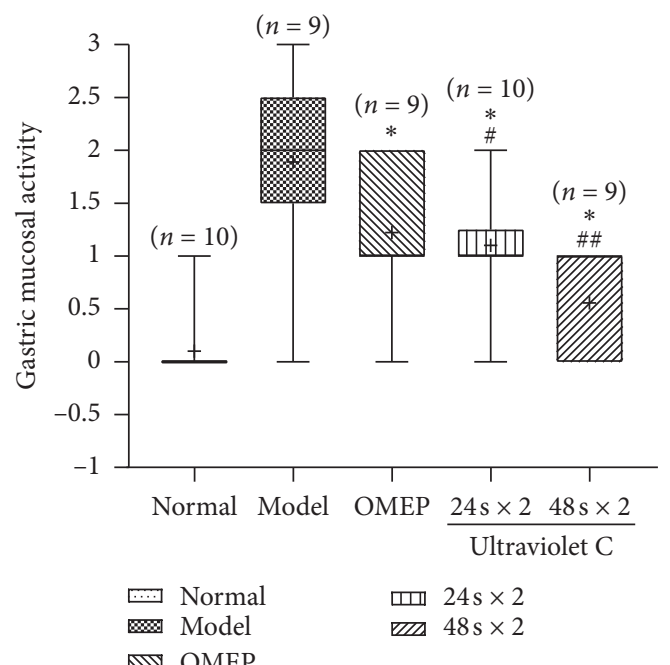

(a)

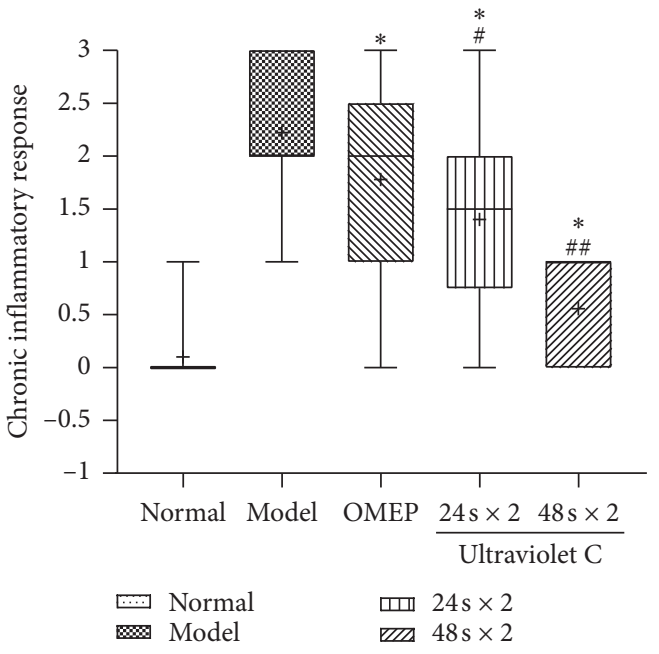

(b)

FIgUre 2: Continued. 


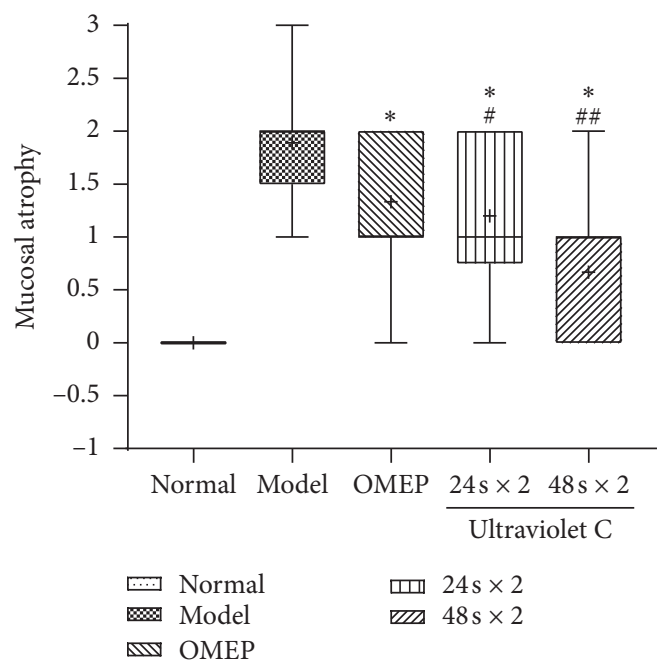

(c)

Figure 2: (a) Gastric mucosal activity. (b) Chronic inflammatory response. (c) Gastric mucosal atrophy. ${ }^{*} P<0.05$ versus model. ${ }^{\#} P<0.05$, ${ }^{\# \#} P<0.01$ versus OMEP, and ${ }^{\# \#} P<0.01$ versus $24 \mathrm{~s} \times 2$.

TABle 2: The degrees of gastric mucosal activity, chronic inflammatory response, and gastric mucosal atrophy (M, QR).

\begin{tabular}{lcccc}
\hline Groups & $n$ & Gastric mucosal activity & Chronic inflammatory response & Gastric mucosal atrophy \\
\hline Normal & 10 & $0.00,0.00$ & $0.00,0.00$ & $0.00,0.00$ \\
Model & 9 & $2.00,1.00$ & $2.00,1.00$ & $2.00,1.00$ \\
OMEP & 9 & $1.00,1.00^{*}$ & $2.00,2.00^{*}$ & $1.00,1.00^{*}$ \\
Ultraviolet C $(24 s \times 2)$ & 10 & $1.00,0.00^{*} \#$ & $1.50,1.00^{*} \#$ & $1.00,1.00^{*} \#$ \\
Ultraviolet C $(48 \mathrm{~s} \times 2)$ & 9 & $1.00,1.00^{*} \# \#$ & $1.00,1.00^{*} \# \#$ & $1.00,1.00^{*} \# \#$ \\
\hline
\end{tabular}

Data are expressed as median, quartile range $(\mathrm{M}, \mathrm{QR}) .{ }^{*} P<0.05$ versus model, ${ }^{\#} P<0.05$ versus OMEP, and ${ }^{\# \#} P<0.05$ versus $24 \mathrm{~s} \times 2$.

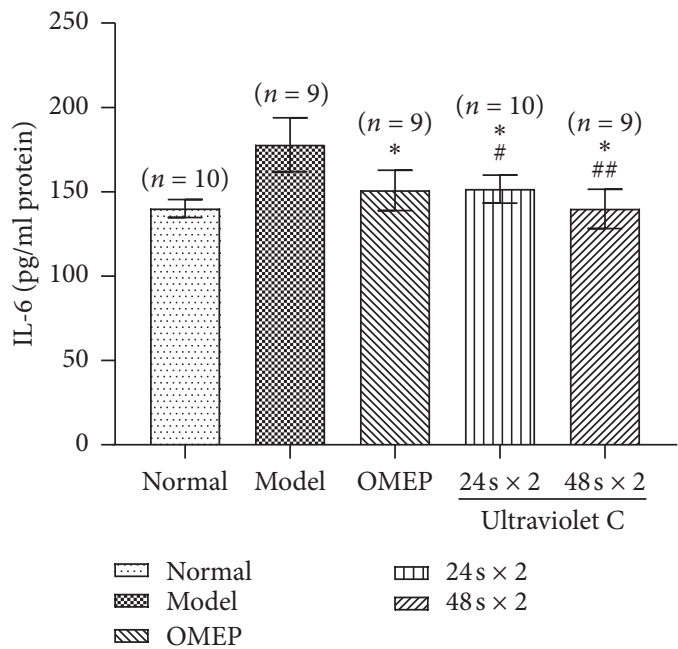

(a)

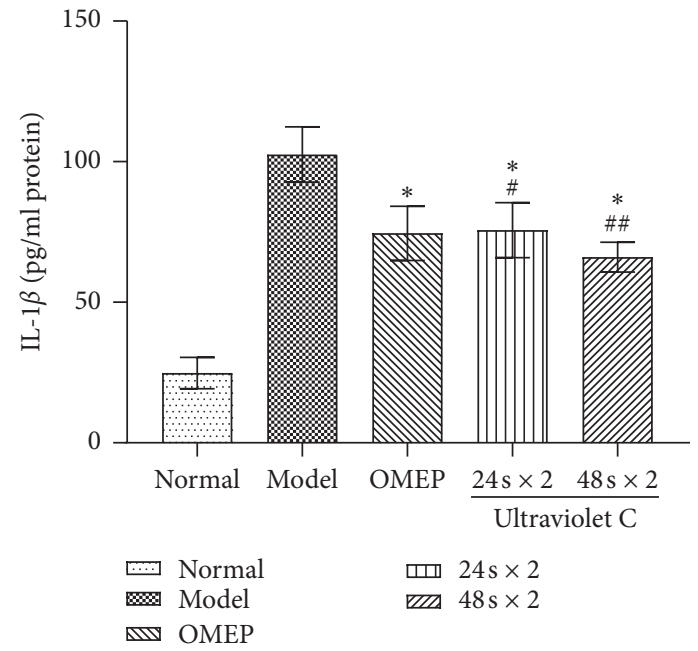

(b)

FIgUre 3: Continued. 


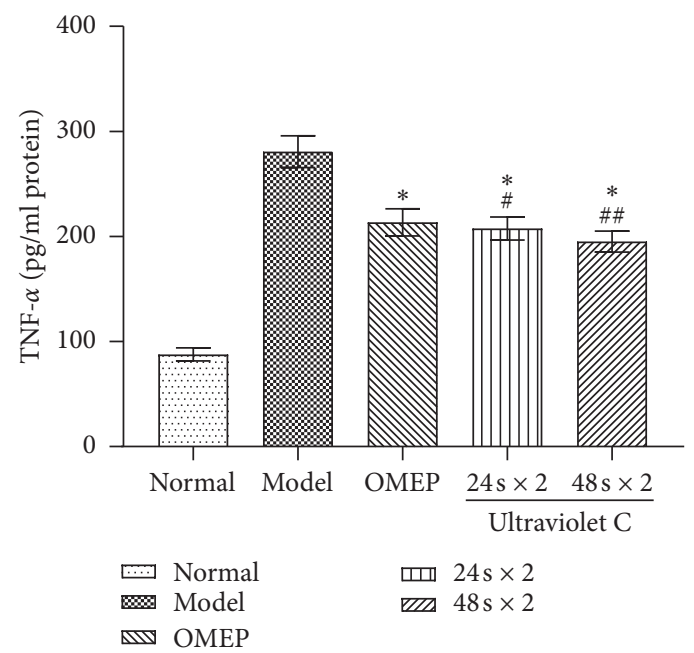

(c)

Figure 3: (a) IL-6. (b) IL-1 $\beta$. (c) TNF- $\alpha .{ }^{*} P<0.05$ versus model. ${ }^{\#} P<0.05,{ }^{\# \#} P<0.05$ versus OMEP, and ${ }^{\# \#} P<0.05$ versus $24 \mathrm{~s} \times 2$.
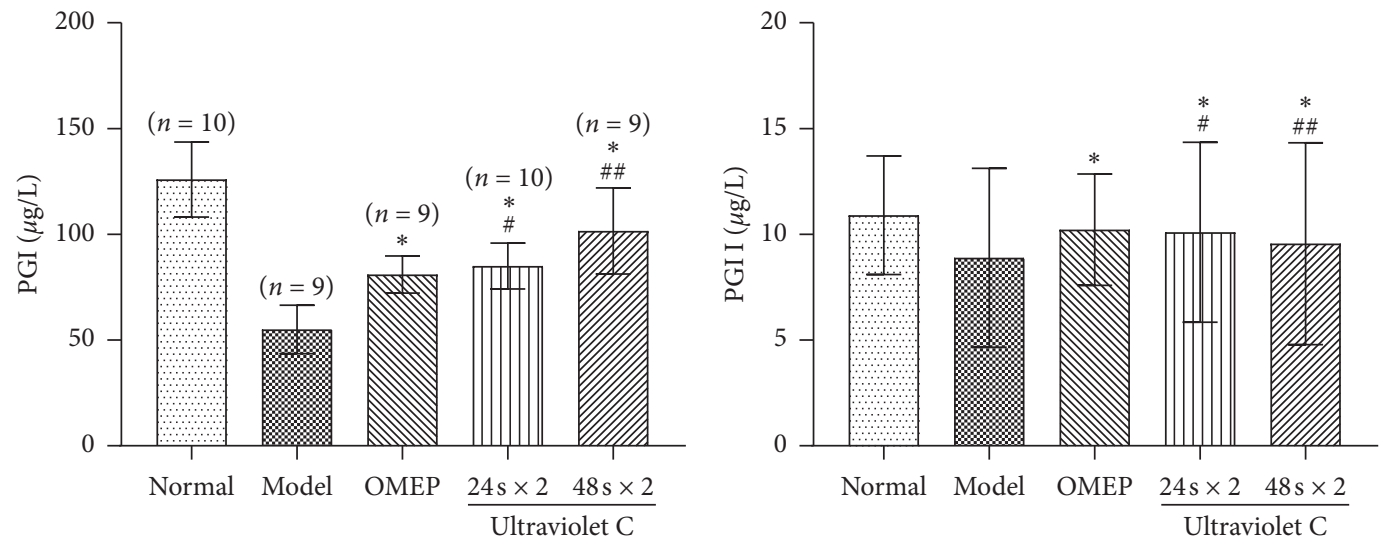

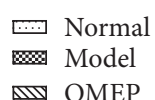

एسل $24 \mathrm{~s} \times 2$

एवा $48 \mathrm{~s} \times 2$

(a)
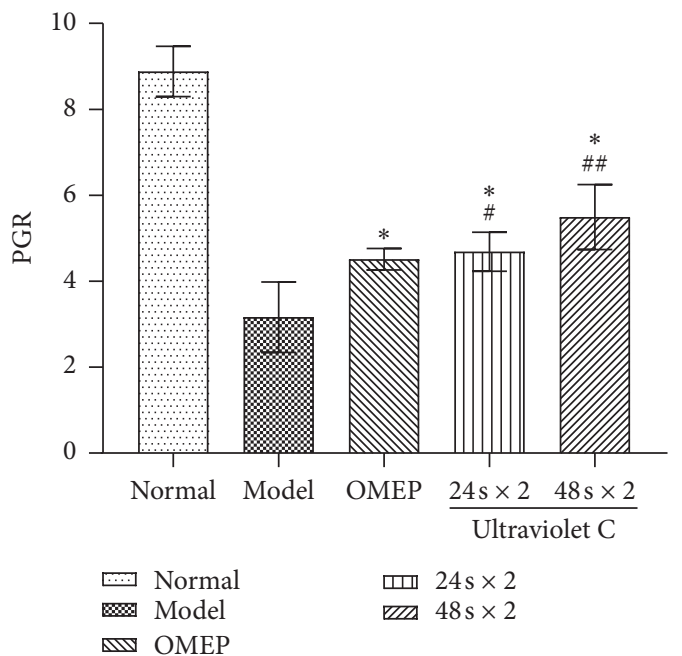

$\ldots$ Normal

Model

WI OMEP
미 $24 \mathrm{~s} \times 2$

चाय $48 \mathrm{~s} \times 2$

(b)

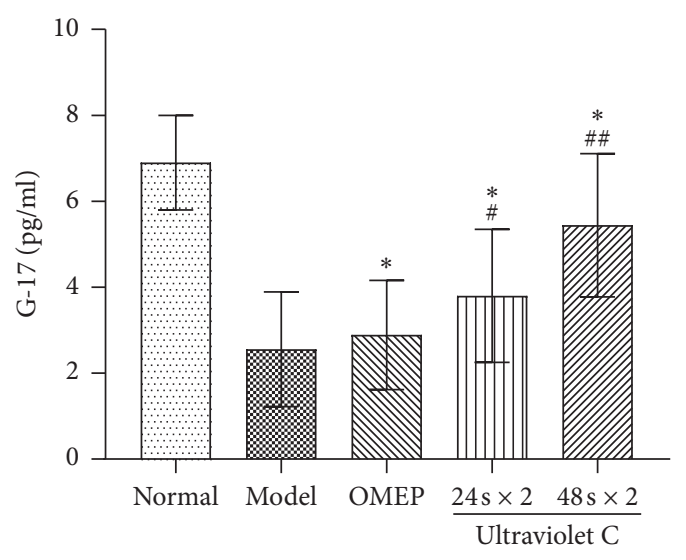

… Normal

Model

OMEP

(c)

(d)

Figure 4: (a) PGI. (b) PGII. (c) PGR. (d) G-17. (a, b, d) ${ }^{*} P<0.01$ versus model, ${ }^{\# \#} P<0.01$ versus $24 \mathrm{~s} \times 2$, and ${ }^{\#} P>0.05$ versus OMEP. (c) ${ }^{*} P>0.05$ versus model, ${ }^{\#} P>0.05$ versus OMEP, and ${ }^{\# \#} P>0.05$ versus $24 \mathrm{~s} \times 2$. 


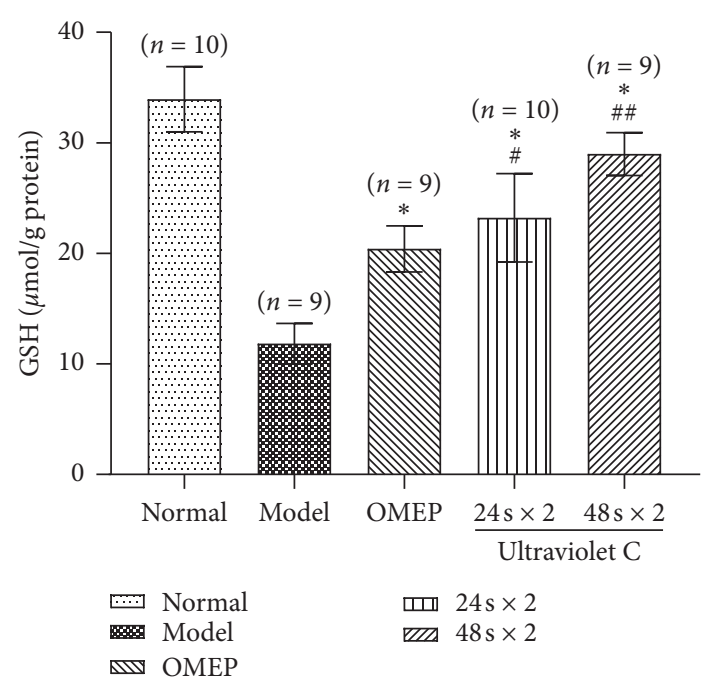

(a)

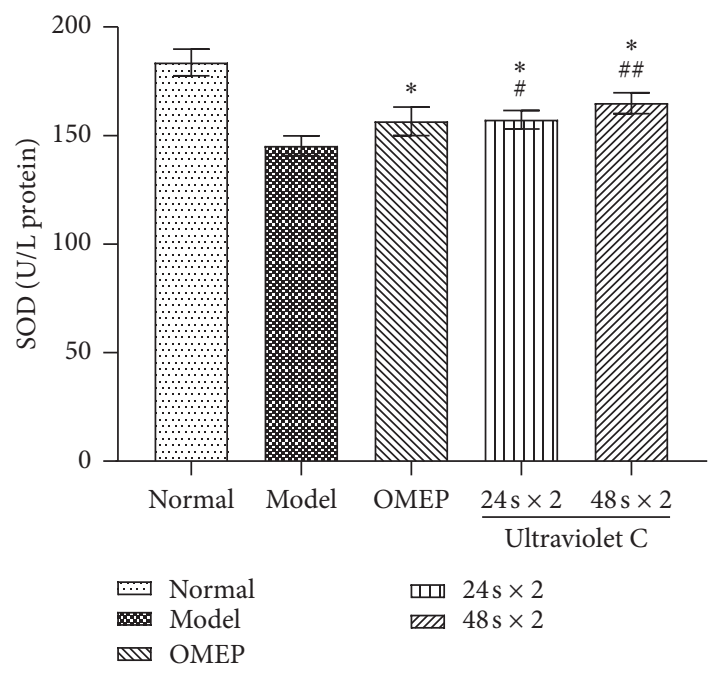

(c)

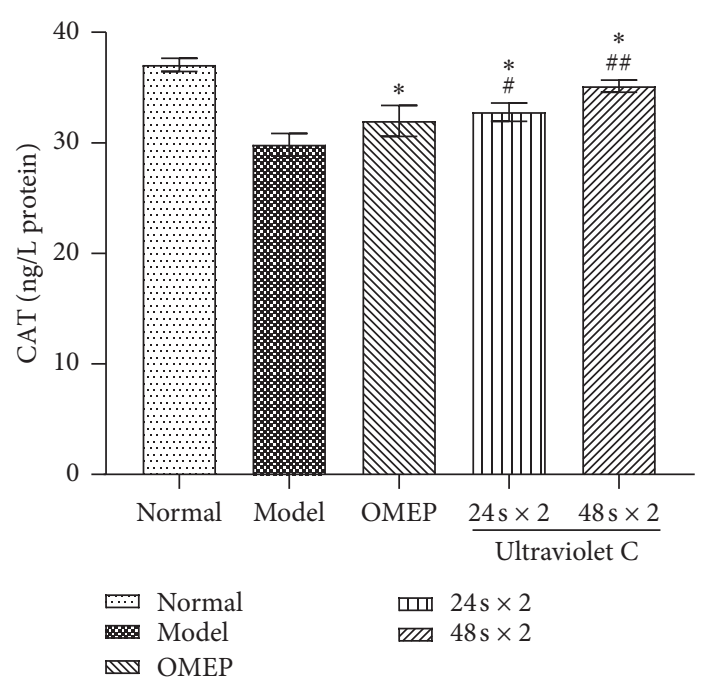

(b)

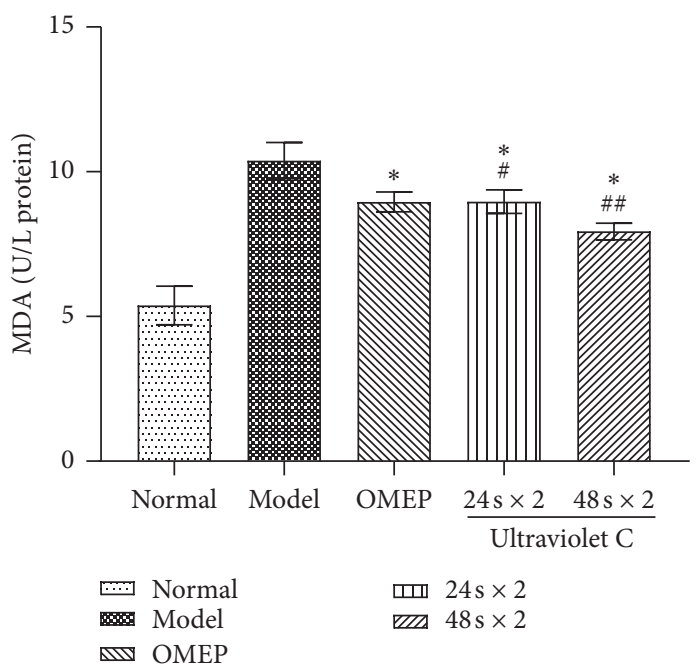

(d)

Figure 5: (a) GSH. (b) CAT. (c) SOD. (d) MDA ${ }^{*} P<0.05$ versus model. ${ }^{\#} P>0.05$ versus OMEP. ${ }^{\# \#} P<0.01$ versus OMEP, $24 \mathrm{~s} \times 2$.

some other cells could exert their anti-inflammatory effects. Moreover, UVC could suppress inflammatory response and reduce inflammation (some CG cases are induced because of autoimmunity disorder) [29-31].

Although the pro-regenerative effects of UVC have been widely reported, almost no researches focused on its effects on chronic gastric mucosal injury. Herein, this study was aimed at investigating the protective effects of UVC on gastric mucosa and the mechanisms.

Previous reports have confirmed that the combination of ethanol, sodium deoxycholate, and ammonium hydroxide could induce the occurrence of CG [16]. Mucosal atrophy and neutrophil infiltration were typical histopathological alternations of CG, which could be used to determine whether the model was established. OMEP is a commonlyused drug for the treatment of CG, which then was selected as the positive drug in this work [32].

IL-6, IL- $1 \beta$, and TNF- $\alpha$, as important proinflammatory mediators, are often used to evaluate the severity of gastric mucosal injury. Previous studies have found that the levels of TNF- $\alpha$ and IL- $1 \beta$ in patients with chronic gastritis are higher than those in normal people, and their levels increase with the aggravation of gastric mucosal lesions, suggesting that these inflammatory factors may play a role in aggravating the inflammatory response to chronic gastritis. In our research, UVC can significantly inhibit the serum IL-6, IL- $1 \beta$, and TNF- $\alpha$ of the model rats, and reduce the inflammatory reaction.

According to the consensus on chronic gastritis in China published in 2017 [19], the levels of serum PGI, PGII, PGR, and G-17 could be used to accurately distinguish the secretion features, atrophy degrees, and tissue function of gastric mucosa in different areas. Thus, the measurement of these markers has been recognized as a reliable noninvasive method for diagnosing gastric mucosal diseases in early stages $[6,33]$. Several previous studies indicated that the levels of PGI and PGR were positively correlated with the severity of corpus gastritis [34-37], while the level of G-17 decreased with the aggravation of antral inflammation and 
atrophy [38-40]. This study demonstrated that UVC irradiation could not only increase the levels of serum PGI and PGR but also increase the G-17 levels. These results suggested that irradiation with UVC of different doses for 2 yields could fully cover gastric corpus and antrum, which further alleviated chronic gastric inflammation and promoted the repair of atrophic mucosa.

In this study, our results suggested that UVC irradiation could increase CAT and SOD activity as well as GSH levels and decrease MDA content to enhance the antioxidative ability of rats with CG induced by physiochemical irritants. These results indicated that UVC irradiation could restore the balance between aggressive and defensive factors by modulating the antioxidative function, which further protected gastric mucosal cells from the attack by free radicals, thereby relieving gastric tissue injury.

\section{Conclusions}

This study showed that UVC irradiation, especially $48 \mathrm{~s} \times 2$ yields, could effectively improve gastric mucosal activity, inflammatory response, and atrophy, inhibit inflammation factor, increase the contents of PGI, PGR, and G-17, improve the antioxidative effect of gastric mucosa, and protect gastric mucosa of rats with CG induced by physiochemical irritants. Our current results suggested that UVC could be considered as a promising novel therapeutic strategy for CG.

\section{Data Availability}

The data used to support the findings of this study are available from the corresponding author upon request.

\section{Conflicts of Interest}

The authors declare no conflicts of interest.

\section{Acknowledgments}

This study was supported by the Clinical Research Support Fund of Chinese PLA General Hospital (2019XXJSYX10).

\section{References}

[1] E. Miceli, A. Vanoli, M. V. Lenti et al., "Natural history of autoimmune atrophic gastritis: a prospective, single centre, long-term experience," Alimentary Pharmacology and Therapeutics, vol. 50, no. 11-12, pp. 1172-1180, 2019.

[2] D. M. McCarthy, "Proton pump inhibitor use, hypergastrinemia, and gastric carcinoids-What is the relationship?" International Journal of Molecular Sciences, vol. 21, no. 2, Article ID E662, 2020.

[3] R. Fossmark, T. C. Martinsen, and H. L. Waldum, "Adverse effects of proton pump inhibitors-evidence and plausibility," International Journal of Molecular Sciences, vol. 20, no. 20, Article ID E5203, 2019.

[4] R. Orel and H. Turk, "Re: might the use of acid-suppressive medications predispose to the development of eosinophilic esophagitis?" American Journal of Gastroenterology, vol. 105, no. 2, p. 468, 2010 author reply 469.
[5] S. N. Merwat and S. J. Spechler, "Might the use of acidsuppressive medications predispose to the development of eosinophilic esophagitis?" The American Journal of Gastroenterology, vol. 104, no. 8, pp. 1897-1902, 2009.

[6] R. Wang and X. Z. Chen, "Prevalence of atrophic gastritis in southwest China and predictive strength of serum gastrin-17: a cross-sectional study (SIGES)," Scientific Reports, vol. 10, no. 1, Article ID 4523, 2020.

[7] K. Nakai, Y. Numaguchi, T. H. Foster, K. Shima, and M. Kikuchi, "Endovascular treatment using low-power ultraviolet laser for delayed vasospasm in the rabbit carotid artery model," AJNR American Journal of Neuroradiology, vol. 23, no. 10, pp. 1725-1731, 2002.

[8] W. Suo, "Effect of ultraviolet C irradiation on expression of basic fib roblast growth factor of wound in rats," Chinese Journal of Physical Medicine and Rehabilitation, vol. 25, no. 11, pp. 651-654, 2003.

[9] W. Suo, A. M. Ma, and X. L. Wang, "Ultraviolet C irradiation induced L-hydroxyproline changes in the gunshot wound tissue of limbs," Journal of Clinical Rehabilitative Tissue Engineering Research, vol. 11, no. 1, pp. 99-102, 2007.

[10] W. Suo, X. L. Wang, and D. W. Wang, "Effect of ultraviolet C irradiation on expression of platelet-derived growth factor in granulation tissues of wounds," Modern Rehabilitation, vol. 5, no. 20, pp. 73-74, 2001.

[11] W. Suo, X. L. Wang, and D. W. Wang, "Effect of ultraviolet C irradiation on expression of transforming growth factor $\beta$ in wound," Chinese Journal of Rehabilitation Theory Practice, vol. 8, no. 1, pp. 5-7, 2002.

[12] L. Fan, J. Zhang, Y. Wang, R. Yang, L. Tao, and M. Yang, "Curative effect of UVC radiation treatment on inflammation of radioactive oral mucosa," Modern Clinical Nursing, vol. 15, no. 3, pp. 26-28, 2016.

[13] F. Li, "Curative effect evaluation of short-wave ultraviolet ray therapy apparatus for oral mucositis of acute leukemia patients," Journal of Clinical Research, vol. 34, no. 10, pp. 1898-1899, 2017.

[14] H. Zeng, H. Y. Qin, S. E. Shen et al., "Experimental study on the immune function of rats' infected wound by ultraviolet $\mathrm{C}$," Chinese Journal of Rehabilitation Medicine, vol. 22, no. 9, pp. 787-789, 2007.

[15] W. Suo, X. L. Wang, and D. W. Wang, "Effect of ultraviolet c radiation on skin wound healing in rats," Chinese Journal of Rehabilitation Medicine, vol. 17, no. 5, pp. 309-310, 2002.

[16] X. Feng and E. Linghu, "Establishment of animal chronic atrophic gastritis model," Journal of Chinese PLA Postgraduate Medical School, vol. 33, no. 6, pp. 668-671, 2012.

[17] C. S. Wang, M. Yang, J. Yu, and L. Min, "Protective effect of benefiting qi and dissipate stasis on gastric mucosa of atrophic gastritis rat models," Journal of Practical Traditional Chinese Internal Medicine, vol. 26, no. 2, pp. 19-20, 2012.

[18] E. Al-Sayed, H. E. Michel, M. A. Khattab, M. El-Shazly, and A. N. Singab, "Protective role of casuarinin from melaleuca leucadendra against ethanol-induced gastric ulcer in rats," Planta Medica, vol. 86, no. 1, pp. 32-44, 2020.

[19] Chinese Society of Gastroenterology, "Consensus on chronic gastritis in China (2017, Shanghai)," Chinese Journal of Gastroenterology, vol. 22, no. 11, pp. 670-687, 2017.

[20] S. Zhao, Y. Li, and C.-J. Wang, "Evolution of consensus for integration of traditional Chinese and western medicine in the diagnosis and treatment of chronic gastritis for nearly 30 years," China Journal of Traditional Chinese Medicine and Pharmacy, vol. 32, no. 10, pp. 4605-4607, 2017. 
[21] J.-Y. Fang, Y. Q. Du, W. Z. Liu et al., "Chinese consensus on chronic gastritis (2017, Shanghai)," Journal of Digestive Diseases, vol. 19, no. 4, pp. 182-203, 2018.

[22] H. Seo, J. Oh, D. Hahn, C.-S. Kwon, J. S. Lee, and J.-S. Kim, "Protective effect of glyceollins in a mouse model of dextran sulfate sodium-induced colitis," Journal of Medicinal Food, vol. 20, no. 11, pp. 1055-1062, 2017.

[23] H. Fu, "The relation between serum pepsinogen, gastrin-17 and chronic gastritis," Journal of Guiyang Medical College, vol. 36, no. 2, pp. 187-189, 2011.

[24] T. Bito, T. Misaki, Y. Yabuta, T. Ishikawa, T. Kawano, and F. Watanabe, "Vitamin B12 deficiency results in severe oxidative stress, leading to memory retention impairment in Caenorhabditis elegans," Redox Biology, vol. 11, pp. 21-29, 2017.

[25] C. S. Bang, J. J. Lee, and G. H. Baik, "Prediction of chronic atrophic gastritis and gastric neoplasms by serum pepsinogen assay: a systematic review and meta-analysis of diagnostic test accuracy," Journal of Clinical Medicine, vol. 8, no. 5, Article ID E657, 2019.

[26] A. Yanaka, "Role of sulforaphane in protection of gastrointestinal tract against $H$. pylori and NSAID-induced oxidative stress," Current Pharmaceutical Design, vol. 23, no. 27, pp. 4066-4075, 2017.

[27] H. Raza, A. John, and S. Benedict, "Acetylsalicylic acid-induced oxidative stress, cell cycle arrest, apoptosis and mitochondrial dysfunction in human hepatoma HepG2 cells," European Journal of Pharmacology, vol. 668, no. 1-2, pp. 1524, 2011.

[28] L. Li, L. Kong, and H. Song, "The therapeutic effect of zerumbone on chronic gastritis via antioxidant mechanisms," Experimental and Therapeutic Medicine, vol. 14, no. 3, pp. 2505-2510, 2017.

[29] M. L. Kripke, "Immunological unresponsiveness induced by ultraviolet radiation," Immunological Reviews, vol. 80, no. 1, pp. 87-102, 1984.

[30] M. L. Kripke, R. M. Thorn, P. H. Lill, C. I. Civin, N. H. Pazmiño, and M. S. Fisher, "Further characterization of immunological unresponsiveness induced in mice by ultraviolet radiation growth and induction of nonultraviolet-induced tumors in ultraviolet-irradiated mice," Transplantation, vol. 28, no. 3, pp. 212-217, 1979.

[31] F. P. Noonan and E. C. De Fabo, "Immunosuppression by ultraviolet B radiation: initiation by urocanic acid," Immunology Today, vol. 13, no. 7, pp. 250-254, 1992.

[32] T. Zhao, Y. Zhang, S. Mu et al., "Protective effects of genipin on ethanol-induced acute gastric injury in mice by inhibiting NLRP3 inflammasome activation," European Journal of Pharmacology, vol. 867, 2020.

[33] I. Gritti, G. Banfi, and G. S. Roi, "Pepsinogens: physiology, pharmacology pathophysiology and exercise," Pharmacological Research, vol. 41, no. 3, pp. 265-281, 2000.

[34] D. J. Etherington and W. H. Taylor, "The pepsins from human gastric mucosal extracts," Biochemical Journal, vol. 118, no. 4, pp. 587-594, 1970.

[35] V. V. Kharitonin, G. T. Chernenko, E. A. Sdobnova, and A. G. Khliabich, "Enzymatic activity and therapeutic action of pepsin $\mathrm{K}$ in chronic gastritis with secretory failure," Klinicheskaia Meditsina, vol. 81, no. 8, pp. 35-37, 2003.

[36] K. Kojima and M. Moriga, "Studies on the pepsinogens of human gastric mucosal extracts," Gastroenterologia Japonica, vol. 13 , no. 6, pp. 421-427, 1978.

[37] W. H. Taylor, "Studies on gastric proteolysis. 3. The secretion of different pepsins by fundic and pyloric glands of the stomach," Biochemical Journal, vol. 71, no. 2, pp. 384-388, 1959.

[38] A. I. A. Al-Ezzy, "Immunopathological and modulatory effects of cag A+ genotype on gastric mucosa, inflammatory response, pepsinogens, and gastrin-17 secretion in Iraqi patients infected with H. pylori," Open Access Macedonian Journal of Medical Sciences, vol. 6, no. 5, pp. 794-802, 2018.

[39] D. H. Parhusip, G. A. Siregar, and L. B. Dairi, "The difference of serum gastrin-17 level based on gastritis severity and Helicobacter Pylori infection," Open Access Macedonian Journal of Medical Sciences, vol. 7, no. 8, pp. 1266-1269, 2019.

[40] E. Goni, M. Venerito, C. Schulz et al., "Influence of laboratory-related and endoscopy-related factors on the assessment of serum pepsinogens and gastrin-17," European Journal of Gastroenterology \& Hepatology, vol. 29, no. 12, pp. 1340-1345, 2017. 